\title{
A Semiautomated Method for Quantification of F 18 Florbetapir PET Images
}

\author{
Abhinay D. Joshi, Michael J. Pontecorvo, Ming Lu, Daniel M. Skovronsky, Mark A. Mintun, and Michael D. Devous, Sr. \\ Avid Radiopharmaceuticals, Inc., Philadelphia, Pennsylvania
}

PET amyloid imaging is increasingly used in research trials related to Alzheimer disease and has potential as a quantitative biomarker. This study had 3 objectives: first, to describe a semiautomated quantitative method that does not require subject-specific MR imaging scans for estimating F 18 Florbetapir plaque binding using 10-min PET images; second, to evaluate the method's accuracy for identifying positive and negative scans; and third, to correlate derived standardized uptake value ratios to neuropathologic measures of amyloid. Methods: The F 18 Florbetapir PET images are initially converted to Montreal Neurologic Institute brain atlas space using an internally developed PET target F 18 Florbetapir template. Subsequently, a single mean cortical standardized uptake value ratio (mcSUVr) is calculated from the mean standardized uptake value of 6 cortical regions normalized to a reference region. Four reference regions were explored: whole cerebellum, cerebellar gray matter, pons, and centrum semiovale. The performance of the resultant mcSUVrs were evaluated in 74 young cognitively normal subjects (age $<50 \mathrm{y}$ ) with a negligible likelihood of amyloid $\beta$ pathology, and in 59 deceased subjects with autopsy-based amyloid $\beta$ neuritic plaque measure who underwent F 18 Florbetapir PET imaging before death. Results: Significant correlations were obtained between mcSUVrs and 3 different pathologic measures of amyloid deposition at autopsy using all 4 reference regions, with the whole-cerebellum mcSUVr correlating most strongly across pathologic measures $(r=0.71-0.75, P<0.0001)$. Using the whole-cerebellum mcSUVr and a threshold mcSUVr of less than 1.10, $100 \%$ of young cognitively normal subjects were correctly classified as amyloid-negative (mcSUVr range, 0.87-1.08). Similarly, 20 of 20 autopsy-negative subjects showed mcSUVrs of 1.10 or less, whereas 38 of 39 pathology-verified amyloid-positive subjects had mcSUVrs of more than 1.10. Conclusion: This semiautomated $\mathrm{F} 18$ Florbetapir PET quantification method yielded mcSUVrs that significantly correlated with measures of amyloid pathology at autopsy. The method also effectively discriminated autopsy-identified amyloidpositive and -negative cases using a whole-cerebellum mcSUVr threshold of 1.10 .

Key Words: Alzheimer's; florbetapir; PET; quantification; neuropathology

J Nucl Med 2015; 56:1736-1741

DOI: 10.2967/jnumed.114.153494

$\mathbf{I}_{\mathrm{n}}$

maging techniques using PET tracers that bind to aggregated amyloid $\beta(\mathrm{A} \beta)$ peptides in amyloid plaques have the potential to

Received Dec. 23, 2014; revision accepted Jul. 9, 2015.

For correspondence or reprints contact: Abhinay D. Joshi, Avid

Radiopharmaceuticals, 3711 Market St., 7th Floor, Philadelphia, PA 19104.

E-mail: joshi@avidrp.com

Published online Sep. 3, 2015.

COPYRIGHT (c) 2015 by the Society of Nuclear Medicine and Molecular Imaging, Inc. assess brain amyloid plaque pathology. Studies using ${ }^{11} \mathrm{C}$-Pittsburgh compound $\mathrm{B}\left({ }^{11} \mathrm{C}-\mathrm{PIB}\right)$ were the first to demonstrate the feasibility of this approach (1). More recently, ${ }^{18} \mathrm{~F}$ ligands have been used for the same purpose $(2,3)$.

In vivo studies have shown that $\mathrm{F} 18$ Florbetapir can image cortical A $\beta$ load in clinically probable Alzheimer disease (AD) patients, patients with mild cognitive impairment, and healthy controls (HCs) (4). The observed pattern is similar to that previously reported for ${ }^{11} \mathrm{C}$-PIB $(5,6)$, and the distribution is consistent with the known localization of amyloid (7). Although F 18 Florbetapir PET standardized uptake value ratio has previously been reported $(4,8)$, the technique that was used to develop the atlas-based volumes of interest (VOIs), create an F 18 Florbetapir PET template, and validate reference regions using the autopsy data has not been described in detail. This report describes this methodology and explores its accuracy in two well-characterized datasets, including one with autopsy-based quantification of amyloid burden.

\section{MATERIALS AND METHODS}

The PET-only semiautomated method for quantification of F 18 Florbetapir PET images comprises 3 steps (Fig. 1). First, images for each subject are converted to Montreal Neurologic Institute (MNI) (9) standard stereotactic brain atlas space using a specialized F 18 Florbetapir PET template. Second, after inspection (and correction if necessary) of spatial normalization, predefined VOIs in the MNI brain atlas are applied to the converted F 18 Florbetapir PET images. Third, values from a single mean cortical standardized uptake value ratio (mcSUVr) are calculated relative to reference regions of choice. The data used in this report were acquired in previous clinical studies $(4,8,10)$, which had institutional review board-approved protocols and obtained written informed consent from each subject enrolled.

\section{Creation of the F 18 Florbetapir PET Template}

To avoid the need for subject-specific MR imaging scans, an F 18 Florbetapir PET template was created. For this purpose, F 18 Florbetapir PET data from 26 subjects ( 11 with clinically diagnosed AD [age range, 55-88 y] and 15 old HCs [age range, 58-86 y]) from a previous clinical trial (10) were used. Dynamic PET brain images were collected for approximately $90 \mathrm{~min}$ over 30 frames $(4 \times$ $0.25 \mathrm{~min}, 4 \times 0.5 \mathrm{~min}, 3 \times 1 \mathrm{~min}, 2 \times 2 \mathrm{~min}, 5 \times 4 \mathrm{~min}$, and $12 \times$ $5 \mathrm{~min})$ after injection of $370 \mathrm{MBq}( \pm 10 \%)$ of $\mathrm{F} 18$ Florbetapir. Using statistical parametric mapping (SPM2) (11), the PET image frames for each subject were realigned to create a mean image across all frames (Supplemental Fig. 1). This mean image was spatially normalized to a PET template image supplied by SPM2 (11) that conforms to MNI space. The resultant transformation matrix was then used to spatially normalize all PET image frames for each subject to MNI space. Normalized images from two 5-min frames beginning at $50 \mathrm{~min}$ after injection were averaged to create a mean 10-min image representative of 


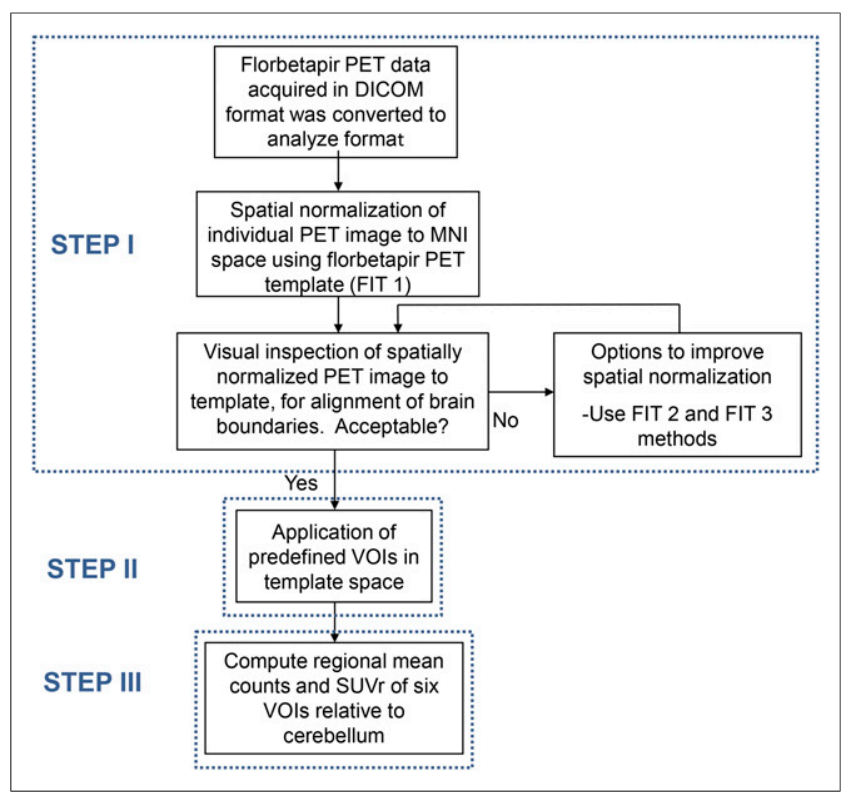

FIGURE 1. Flowchart of semiautomated quantification method applied to F 18 Florbetapir PET scan. There are 3 important steps of semiautomated quantification: spatial normalization of subject data to $\mathrm{MNI}$ space, application of predefined atlas space VOls to transformed subject image, and calculation of mcSUVrs.

the 40- to 90 -min asymptotic period for this tracer (10). These 10-min images were then averaged across all 26 subjects to form the F 18 Florbetapir PET template (Supplemental Fig. 1).

\section{VOI Delineation}

VOIs that represented only gray matter with a high likelihood of having elevated F 18 Florbetapir uptake in $A \beta$-positive subjects were delineated anatomically but were refined using an unbiased approach with information from 3 datasets: voxels representing subject-specific gray matter from MR imaging scans transformed into MNI space; voxels with a high probability of gray matter content based on high regional cerebral blood flow (F 18 Florbetapir uptake in the first few minutes after injection); and voxels identified as having elevated F 18 Florbetapir uptake by a voxelwise comparison of PET images of clinically diagnosed AD subjects with old HCs. These 3 datasets were used as visual guides to refine anatomically defined VOIs from the Talairach atlas.

Subject MR images were spatially normalized using the standard T1-weighted MR imaging template in SPM2. These images were then segmented into gray matter, white matter, and cerebrospinal fluid using SPM2 (Fig. 2A, top row). The gray matter-segmented image was used to create a gray matter binary map.

For $15 \mathrm{HCs}$, an early perfusion image was created as an average of dynamic frames from the first $10 \mathrm{~min}$ after $\mathrm{F} 18$ Florbetapir administration. The average gray matter perfusion image was then masked using the binary MR imaging gray matter map, yielding a high perfusion-specific gray matter map (Fig. 2A, middle row).

Biologically relevant voxels that typically have high amyloid retention within the VOIs were identified via a 2-sample $t$ test (SPM2) comparing 11 AD subjects with 15 HC subjects. F 18 Florbetapir PET images normalized to cerebellar gray matter were used for the $t$ test. No proportional scaling or grand mean scaling was performed, and a relative threshold masking at $80 \%$ of mean voxel values was used. Statistical significance was tested using an extent threshold of zero voxels and an uncorrected threshold of $P<0.05$ to include all relevant clusters and voxels. Figure 3 shows the resultant distribution of significant voxels. The greatest uptake in AD patients relative to HCs was predominantly in the lateral frontal, medial orbital frontal, parietal temporal, and occipital lobes and in the anterior cingulate, posterior cingulate, and precuneus. The centrum semiovale, pons, and cerebellum regions were spared.

Finally, VOIs defined by the atlas were edited by visual inspection using the gray matter, regional cerebral blood flow, and SPM2 masks such that voxels within atlas-defined regions but not represented in the 3 masks were removed. The anatomic location of voxels within the 3 masks was determined using a database from the Talairach Daemon website, which provides coordinates for voxels within anatomic components of the Talairach atlas $(12,13)$. It was necessary to convert Talairach coordinates from the Daemon to MNI space using the MATLAB (The MathWorks) function tal2icbm transform (14). In addition, the lateral $2 \mathrm{~mm}$ of each VOI were removed to limit partialvolume intrusion of extracerebral cerebrospinal fluid into the VOIs.

This process resulted in 17 VOIs in the cortical gray matter (medial orbital frontal, lateral temporal, precuneus, anterior cingulate, posterior cingulate, lateral parietal, and lateral occipital), striatum (left and right anterior putamen, left and right posterior putamen, and left and right caudate), white matter (centrum semiovale and pons), and cerebellum (cerebellar gray matter, entire cerebellum, and cerebellar white). However, the SPM2 analysis of 11 AD subjects acquired as part of a previous study (10) identified higher accumulation of F 18 Florbetapir in 6 of these regions (medial orbital frontal, temporal, parietal, anterior cingulate, posterior cingulate, and precuneus), which were also expected to have high accumulation of $A \beta$ based on the neuropathology literature. Thus, these 6 regions were selected for inclusion in the mcSUVr VOI and are displayed in the top 3 rows of Figure 2B. The final VOIs were similar to those used in earlier studies $(5,7)$.

\section{Semiautomated Method of Quantification}

As shown in Figure 1, the F 18 Florbetapir PET template (Supplemental Fig. 1) and the 6 defined VOIs (Fig. 2B, top 3 rows) were then used in a semiautomated method for extraction of regional mcSUVrs. This method includes spatial normalization of PET images to the F 18 Florbetapir PET template using SPM2 (using default values for all parameters except adding heavy regularization), visual examination of spatial normalization results, application of predefined VOIs, and calculation of mcSUVrs. The spatial normalization results were visually examined for alignment of the patient brain to the template brain, and

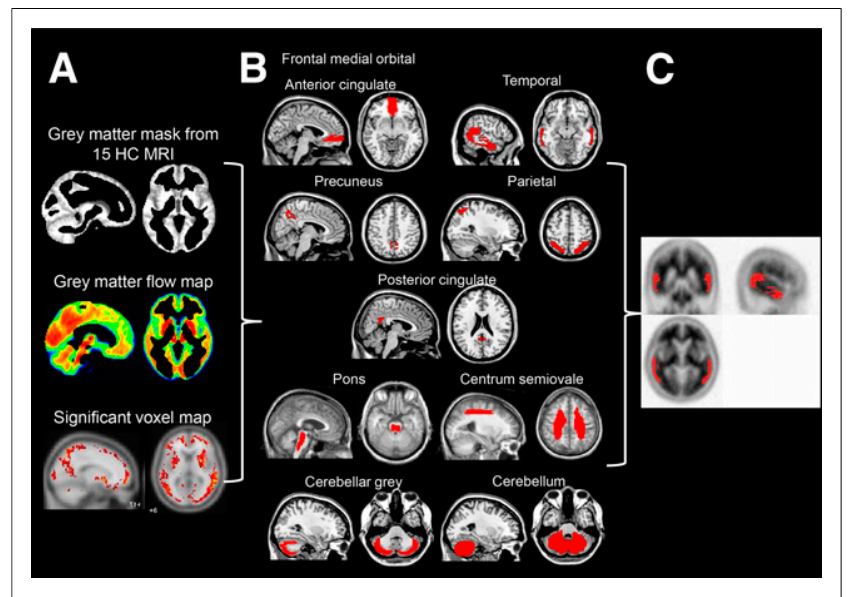

FIGURE 2. (A) Data used to create VOls. (B) VOls (red) for 6 cortical regions and 4 reference regions overlaid on MR template images in $\mathrm{MNI}$ space. First image in top row shows overlay of frontal medial orbital and anterior cingulate regions; only 1 region is seen because of overlap in 2 VOls. (C) Example of temporal VOI overlaid on F 18 Florbetapir PET template. 


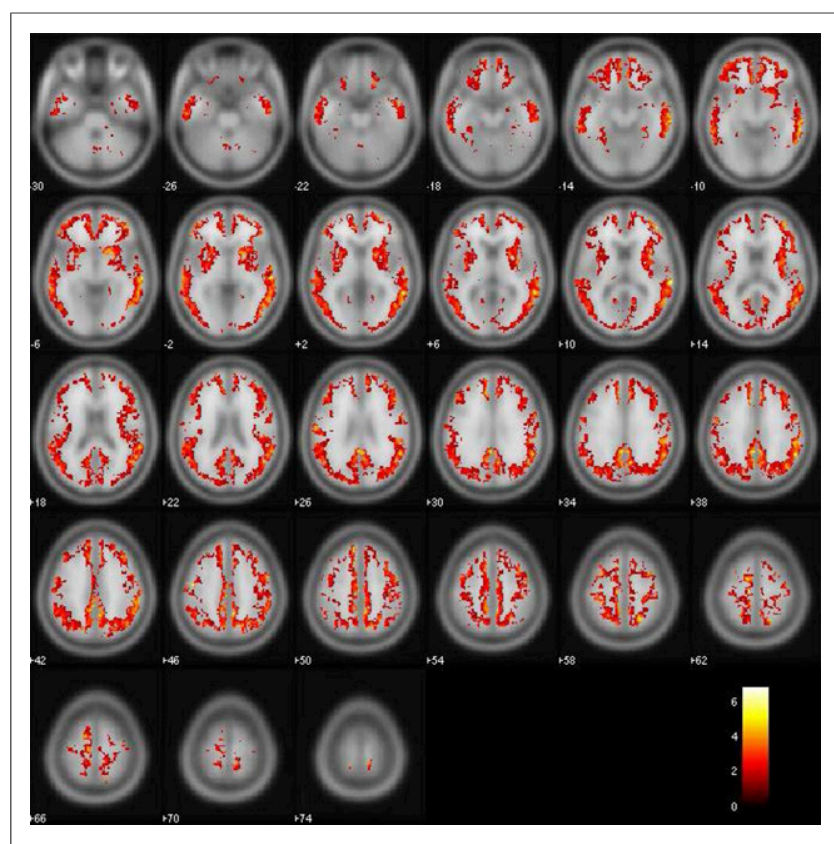

FIGURE 3. Significant voxels for which F 18 Florbetapir retention in clinically diagnosed $\operatorname{AD}(n=11)$ was greater than that in HC subjects $(n=15)$, with uncorrected threshold of $P<0.05$.

cases that were adequately fit were referred to as successful via fit 1 . Cases with unacceptable results ( $>2 \mathrm{~mm}$ offset in the alignment) from the default spatial normalization (fit 1) were spatially normalized using a brain mask weighting factor; this method is referred to as fit 2 . The use of a brain mask provides a guide to limit spatial normalization of the patient's brain to the template brain instead of the patient's head to the template head. It reduces normalization errors caused by extracranial activities such as counts in the thick skull or thick layer of meninges. Cases with unacceptable results from fit 2 were spatially normalized by first using the brain extraction tool (15) in MRIcro (16) to remove the skull from the patient image and then fitting that image and applying the transformation matrix to the original image (fit 3). All scans were successfully fit with 1 of these 3 methods done in sequence. After acceptable spatial normalization, the transformed images were then used for mcSUVr calculation. Regional mean counts were calculated using the 6 selected cortical gray matter regions (medial orbital frontal, temporal, anterior cingulate, posterior cingulate, parietal, and precuneus). The ratio of mean counts from these 6 regions with respect to the mean uptake in a reference region was combined to represent the mcSUVr. Separate mcSUVrs were determined for each reference region (whole cerebellum, cerebellar gray matter, centrum semiovale, and pons; Fig. 2B, bottom 2 rows).

\section{Correlation with Amyloid Burden and Selection of a Reference Region}

The utility of the semiautomated method was assessed by evaluating whether mcSUVrs could be used to separate young HCs from subjects with $\mathrm{AD}$ or mild cognitive impairment and by image-toautopsy correlation (4). Two sets of subjects were analyzed from previously published studies $(4,8)$ : 74 young cognitively normal subjects (YCNs) (age range, 18-50 y; mini-mental state examination [MMSE] score, 29-30) who by age and absence of risk factors should have had no $\mathrm{A} \beta$, and 59 subjects who subsequently died and were autopsied (age range, 47-103 y; MMSE, 0-30). These autopsy-cohort subjects have been previously described (8) and were classified clinically (Table 1$)$ as $\mathrm{AD}(n=29)$, other dementing disorder $(n=13)$, cognitively normal $\mathrm{HC}$ with no history of cognitive impairment or dementia $(n=12)$, or mild cognitive impairment $(n=5)$.

The evaluation of the autopsy results of the 59 participants has been described previously $(4,8): 39$ of 59 subjects were judged positive for A $\beta$ (pathology-positive), whereas 20 of 59 were considered negative for $\mathrm{A} \beta$ (pathology-negative) according to the neuropathologist's diagnosis. For the correlation analyses in this report, autopsy assessments included classification of $A \beta$ neuritic plaque frequency using a 4-point semiquantitative CERAD (Consortium to Establish a Registry for Alzheimer's Disease) scale (0, none; 1, sparse; 2, moderate; 3, frequent neuritic plaques) (17) and quantification of total cortical amyloid burden using $A \beta$ antibody immunohistochemistry (4).

TABLE 1

mcSUVr, Age, and MMSE for YCN and 59 Autopsy Cases

\begin{tabular}{|c|c|c|c|c|c|c|c|}
\hline \multirow[b]{2}{*}{ Parameter } & \multirow[b]{2}{*}{ YCN } & \multicolumn{6}{|c|}{59 autopsy cases } \\
\hline & & $A D$ & ODD & $\mathrm{MCl}$ & $\mathrm{OCN}$ & Pathology-positive & Pathology-negative \\
\hline$n$ & 74 & 29 & 13 & 5 & 12 & 39 & 20 \\
\hline Age (y) & $27 \pm 6$ & $81.0 \pm 11$ & $79 \pm 11$ & $69 \pm 18$ & $80 \pm 14$ & $80 \pm 11^{\star}$ & $78 \pm 16$ \\
\hline MMSE & $29.7 \pm 0.6$ & $12 \pm 7$ & $9 \pm 9$ & $25 \pm 3$ & $26 \pm 5$ & $12 \pm 8^{\star \dagger}$ & $23 \pm 8$ \\
\hline $\begin{array}{c}\text { mcSUVr relative to... } \\
\text { Entire cerebellum }\end{array}$ & $0.96 \pm 0.04$ & $1.39 \pm 0.26$ & $1.18 \pm 0.33$ & $1.04 \pm 0.10$ & $0.98 \pm 0.09$ & $1.38 \pm 0.25^{\star \dagger}$ & $0.94 \pm 0.09$ \\
\hline $\begin{array}{l}\text { Cerebellar gray } \\
\text { matter }\end{array}$ & $1.13 \pm 0.09$ & $1.63 \pm 0.37$ & $1.37 \pm 0.45$ & $1.19 \pm 0.11$ & $1.13 \pm 0.09$ & $1.61 \pm 0.36^{\star \dagger}$ & $1.09 \pm 0.13$ \\
\hline Pons & $0.67 \pm 0.04$ & $0.91 \pm 0.14$ & $0.79 \pm 0.14$ & $0.70 \pm 0.11$ & $0.68 \pm 0.09$ & $0.90 \pm 0.12^{\star \dagger}$ & $0.65 \pm 0.07$ \\
\hline Centrum semiovale & $0.65 \pm 0.04$ & $0.87 \pm 0.13$ & $0.77 \pm 0.14$ & $0.61 \pm 0.10$ & $0.64 \pm 0.10$ & $0.86 \pm 0.12^{\star \dagger}$ & $0.62 \pm 0.09$ \\
\hline
\end{tabular}

${ }^{\star} P<0.01$ from 2-group $t$ test comparing equality of means between pathology-positive and YCN groups.

${ }^{\dagger} P<0.01$ from 2-group $t$ test comparing equality of means between pathology-positive and -negative groups.

$\mathrm{ODD}=$ other dementing disorder; $\mathrm{MCl}=$ mild cognitive impairment.

Identical VOIs for target and reference regions were used to calculate mcSUVrs in all subjects. Key comparisons of interest are between pathology-positive subjects and -negative subjects and between pathology-positive and YCN subjects. For reference, demographic and mcSUVr data are also provided for 59 autopsy cases classified by clinical diagnosis. Except for age, data are mean \pm SD. 
TABLE 2

Relationship Between mcSUVr and Pathology Data

\begin{tabular}{lcccc}
\hline \multirow{2}{*}{ Autopsy measure } & \multicolumn{3}{c}{ mcSUVr } \\
\cline { 2 - 5 } & Whole cerebellum & Cerebellar gray matter & Pons & Centrum semiovale \\
\hline Global highest neuritic plaque count & 0.71 & 0.68 & 0.68 & 0.71 \\
Immunohistochemistry A $\beta$ burden & 0.75 & 0.71 & 0.72 & 0.70 \\
CERAD score (0- to 3-point scale) & 0.73 & 0.70 & 0.71 & 0.73 \\
AUC and 95\% confidence interval & $0.996(0.988-1.000)$ & $0.980(0.953-1.000)$ & $0.965(0.921-1.000)$ & $0.951(0.904-0.999)$ \\
Cohen d (weighted variance) & 2.77 & 2.37 & 2.83 & 2.24 \\
Coefficient of variance from 74 YCNs & 0.042 & 0.079 & 0.059 & 0.053
\end{tabular}

Data are Spearman rank correlations between mcSUVr and autopsy measures. All correlations were significant $(P<0.0001)$. Cohen $d$ was calculated using weighted pooled variance estimation from pathology-positive and -negative groups as classified on autopsy measures. AUCs were calculated from ROC analyses intended to discriminate pathology-positive and -negative groups using 4 mcSUVr reference regions (Supplemental Fig. 2)

F 18 Florbetapir PET mcSUVrs were calculated using 4 reference regions (entire cerebellum, cerebellar gray matter, centrum semiovale, and pons). For each reference region, $\mathrm{mcSUVr}$ for the 59 autopsy cases was compared with CERAD and immunohistochemistry score using the Spearman correlation coefficient. Effect size (Cohen's d) was calculated for each reference region, comparing cases classified as pathologypositive with those classified as pathology-negative. The difference between group mcSUVrs normalized to the pooled SD of the 2 groups was used to calculate the effect size. Receiver-operating-characteristic (ROC) analyses were also used to evaluate the performance of the different reference regions in classifying cases as pathology-positive and -negative. An area under the curve (AUC) was calculated from the ROC curves. The optimal thresholds (cutoffs) for these ROC curves were derived using the Youden index method (18). From the 4 regions tested, a preferred reference region for calculating the mcSUVr was selected on the basis of the combined results of the correlation analysis, effect size calculation, and AUC estimate.

\section{RESULTS}

All 133 F 18 Florbetapir PET scans, including 74 YCNs and 59 autopsy cases, were successfully spatially normalized to MNI space. Of these 133 cases, 97 were spatially normalized using the default fit 1 method; 29, using the brain mask (fit 2) option; and 7 , using the fit 3 option.

The mcSUVrs calculated by reference region for the 74 YCNs and the 59 autopsy cases (classified by clinical diagnosis and by autopsy measures as pathology-positive or -negative) are listed in Table 1 . The mean values for age, MMSE, and mcSUVr for all 4 reference regions differed significantly between the 74 YCNs and the 39 pathology-positive subjects $(P<0.01)$. The mean MMSE and mcSUVrs for all 4 reference regions also differed significantly between the pathology-positive and -negative subjects $(P<0.01)$.

For all 4 reference regions, significant correlations $(P<0.0001)$ were observed between mcSUVr and all autopsy measures (global highest neuritic plaque count, immunohistochemistry, and CERAD score; Table 2). Comparatively, the whole cerebellum provided the highest correlation with all 3 autopsy measures, ranging from 0.71 to 0.75 . AUC calculations (Table 2; Supplemental Fig. 2) also suggested marginally better classification between the 2 groups using the whole cerebellum as a reference region (0.996 confidence interval, 0.988-1.00). Further, whole-cerebellum
mcSUVr showed the least variability (smallest coefficient of variation) among YCNs, in whom the absence of amyloid pathology would be expected. Effect size estimates (Cohen's d) showed a clear separation $(\mathrm{d}>0.8)$ between pathology-positive and -negative subjects using all reference regions. The largest effect size was achieved for $\mathrm{mcSUVr}$ with pons $(\mathrm{d}=2.83)$ as a reference region, followed by whole cerebellum $(\mathrm{d}=2.77)$.

The distribution of mcSUVrs across the 4 reference regions is illustrated in Figure 4. The threshold lines in Figure 4 indicate the mcSUVr threshold that best classified positive and negative cases obtained from the ROC analysis (1.10 for whole cerebellum, 1.28 for cerebellar gray matter, 0.78 for pons, and 0.68 for the centrum semiovale). The threshold of 1.10 determined on the basis of the ROC analysis in this study is identical to the threshold selected for no detectable amyloid in a prior study (19), based on the upper limit of the 2-sided $95 \%$ confidence interval for YCNs. Using the whole cerebellum as a reference region, there was clear classification with no overlap between the 74 YCN/20 pathologynegative group (black and blue dots) and 39 pathology-positive subjects (red dots) except for a single pathology-positive case, clinically diagnosed as AD, quantified with a mcSUVr of 1.02 . For the other reference regions the classification was not as good, with the scatterplots showing greater overlap between the YCN/ pathology-negative group (black and blue dots) and pathologypositive subjects (red dots) with respect to the optimal classification threshold.

\section{DISCUSSION}

This communication describes a semiautomated method for quantification of F 18 Florbetapir PET data that involved creation of a F 18 Florbetapir PET template in MNI space; VOI delineation based on combined information from PET perfusion maps, anatomic landmarks from corresponding MR imaging scans, and statistical parametric maps distinguishing diagnostic groups; reference region selection based on accuracy in discriminating autopsyconfirmed amyloid-positive from -negative subjects; and correlation with autopsy-based amyloid burden.

The F 18 Florbetapir PET template was based on a ligand-specific template (20) in which F 18 Florbetapir PET images, spatially normalized in MNI space, were averaged from $15 \mathrm{HC}$ subjects and 11 


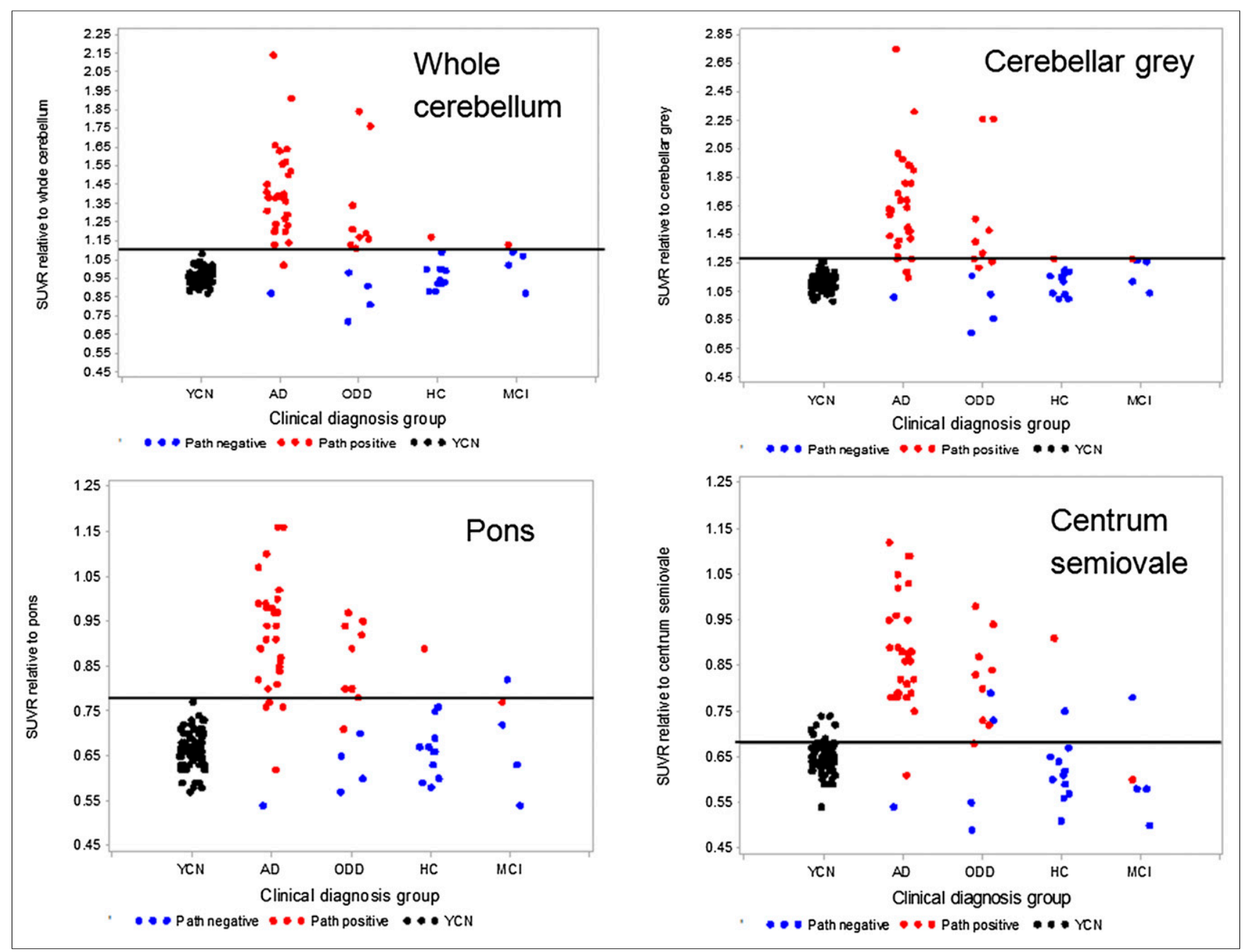

FIGURE 4. Distribution of mcSUVrs (using 4 reference regions) in $74 \mathrm{YCN}$ subjects and in 59 subjects classified by clinical diagnosis who underwent autopsy within 2 y of $F 18$ Florbetapir imaging. Included is best estimated threshold (line) for separation of pathology-positive and -negative subjects from $\mathrm{ROC}$ analysis for whole cerebellum, cerebellar gray matter, pons, and centrum semiovale. Threshold from ROC analysis for whole cerebellum is identical to prespecified threshold in previous trial $(4,8)$.

AD patients (10). No anatomic information from MR imaging or CT was used for spatial normalization or template creation. Edison et al. (21) previously demonstrated that MR imaging and PET template-based spatial normalization methods can produce comparable ${ }^{11} \mathrm{C}$-PIB quantitative results. Similarly Landau et al. (22) have shown a consistently high correlation between ${ }^{11} \mathrm{C}-\mathrm{PIB}$ and $\mathrm{F} 18$ Florbetapir PET scan quantitation from the same patient across a range of different analytic methods that included both an MR imaging-based approach (Freesurfer) and the semiautomated PET template method described in this report. These findings further suggest that the PET template method can provide reliable quantitative estimates even when MR imaging is not available or feasible. The proposed processing stream successfully fit $100 \%$ of the cases in the study. However, technical review was required, and intervention or alternative fit parameters were used in 36 of 133 cases $(27 \%)$.

A unique approach was also followed in creating VOIs, including the use of anatomic information from patient MR images, statistical parametric maps that distinguished clinically diagnosed groups, and early F 18 Florbetapir uptake information representing cerebral perfusion. This approach provided gray matter VOIs that overlapped with MR imaging-defined gray matter segmentation, high perfusion, and significant uptake of F 18 Florbetapir in AD patients as compared with control subjects. The regions of high F 18 Florbetapir uptake identified here are similar to those previously reported for ${ }^{11} \mathrm{C}$-PIB (23) and are also consistent with the distribution of amyloid reported in postmortem studies (7). By designing VOIs to focus on those regions that best differentiate patients with high amyloid burden from those with low burden, we potentially increased the diagnostic sensitivity and specificity of the quantitative technique. However, it remains to be seen whether these regions are the most appropriate for evaluating changes in amyloid, as a result of either increasing disease severity or decreasing amyloid burden with therapy.

Four potential reference regions were considered for calculation of mcSUVr, all of which were expected to have low levels of neuritic amyloid plaques and thus able to adequately estimate nonspecific tracer accumulation. The selection of a standard reference region was informed by obtaining the mcSUVr using these 4 regions relative to amyloid pathology at autopsy. The mcSUVrs for all reference regions tested showed high correlations with amyloid pathology, as measured by both neuritic plaque 
counts and immunohistochemistry. However, slightly higher correlations were obtained using the whole cerebellum than using the other reference regions. Moreover, the whole-cerebellum mcSUVr generated the highest AUC by ROC analysis for classifying pathology-positive and -negative cases (least overlap between YCN/pathology-negative and pathology-positive cases), was comparable to the pons mcSUVr for the effect size difference between pathology-positive and -negative cases, and showed the smallest coefficient of variation among YCNs. Although these differences among reference regions were small, the consistent trend for superior performance with the whole-cerebellum region supports the choice of whole cerebellum as the preferred reference region.

\section{CONCLUSION}

Use of the whole cerebellum as the mcSUVr reference region with an mcSUVr cutoff of 1.10 achieved excellent group separation: $100 \%$ of clinically diagnosed YCN subjects and $100 \%$ of pathologynegative subjects were quantified below the threshold of 1.10 and were successfully classified as $A \beta$-negative, whereas $97.4 \%$ of cases that were considered $\mathrm{A} \beta$-positive by pathologic assessment had an mcSUVr of more than 1.10. Together, these results suggest that an automated quantitation process might be possible and could be a valuable aid in scan interpretation. The present VOIs and reference regions performed accurately and are well suited as a research tool. However, a more fully automated process that does not require expert technical evaluation would be desirable for clinical application.

\section{DISCLOSURE}

The costs of publication of this article were defrayed in part by the payment of page charges. Therefore, and solely to indicate this fact, this article is hereby marked "advertisement" in accordance with 18 USC section 1734. This study was funded by Avid Radiopharmaceuticals, Inc., a wholly owned subsidiary of Eli Lilly and Company, and the authors are employees of Avid Radiopharmaceuticals. No other potential conflict of interest relevant to this article was reported.

\section{REFERENCES}

1. Klunk WE, Wang Y, Huang GF, Debnath ML, Holt DP, Mathis CA. Uncharged thioflavin-T derivatives bind to amyloid-beta protein with high affinity and readily enter the brain. Life Sci. 2001;69:1471-1484.

2. Barthel H, Gertz HJ, Dresel S, et al. Cerebral amyloid- $\beta$ PET with florbetaben $\left({ }^{18} \mathrm{~F}\right)$ in patients with Alzheimer's disease and healthy controls: a multicenter phase 2 diagnostic study. Lancet Neurol. 2011;10:424-435.
3. Rowe CC, Ackerman U, Browne W, et al. Imaging of amyloid $\beta$ in Alzheimer's disease with ${ }^{18}$ F-BAY94-9172, a novel PET tracer: proof of mechanism. Lancet Neurol. 2008;7:129-135.

4. Clark CM, Schneider JA, Bedell BJ, et al. Use of florbetapir-PET for imaging $\beta$-amyloid pathology. JAMA. 2011;305:275-283.

5. Lopresti BJ, Klunk WE, Mathis CA, et al. Simplified quantification of Pittsburgh compound B amyloid imaging PET studies: a comparative analysis. J Nucl Med. 2005;46:1959-1972.

6. Mintun MA, Larossa GN, Sheline YI, et al. $\left[{ }^{11} \mathrm{C}\right]$ PIB in a nondemented population: potential antecedent marker of Alzheimer disease. Neurology. 2006;67: 446-452.

7. Braak H, Braak E. Neuro-pathological staging of Alzheimer-related changes. Acta Neuropathol (Berl). 1991;82:239-259.

8. Clark CM, Pontecorvo MJ, Skovronsky DM, et al. Cerebral PET with florbetapir compared with neuropathology at autopsy for detection of neuritic amyloid- $\beta$ plaques: a prospective cohort study. Lancet Neurol. 2012;11:669-678.

9. Evans AC, Marrett S, Bub D, et al. Anatomical mapping of functional activation in stereotactic coordinate space. Neuroimage. 1992;1:43-53.

10. Wong DF, Rosenberg PB, Zhou Y, et al. In vivo imaging of amyloid deposition in Alzheimer's disease using the novel radioligand ${ }^{18} \mathrm{~F}-\mathrm{AV}-45$ (florbetapir F 18). J Nucl Med. 2010;51:913-920.

11. Friston KJ, Ashburner JT, Kiebel SJ, Nichols TE, Penny WD, eds. Statistical Parametric Mapping: The Analysis of Functional Brain Images. London, U.K.: Academic Press; 2007:10-31.

12. Lancaster JL, Woldorff MG, Parsons LM, et al. Automated Talairach atlas labels for functional brain mapping. Hum Brain Mapp. 2000;10:120-131.

13. Lancaster JL, Rainey LH, Summerlin JL, et al. Automated labeling of the human brain: a preliminary report on the development and evaluation of a forwardtransform method. Hum Brain Mapp. 1997;5:238-242.

14. Lancaster JL, Tordesillas-Gutierrez D, Martinez M, et al. Bias between MNI and Talairach coordinates analyzed using the ICBM-152 brain template. Hum Brain Mapp. 2007;28:1194-1205.

15. Smith SM. Fast robust automated brain extraction. Hum Brain Mapp. 2002;17: $143-155$.

16. Rorden C, Brett M. Stereotaxic display of brain lesions. Behav Neurol. 2000;12:191-200.

17. Mirra SS, Heyman A, McKeel D, et al. The consortium to establish a registry for Alzheimer's disease (CERAD). Neurology. 1991;41:479-486.

18. Youden WJ. Index for rating diagnostic tests. Cancer. 1950;3:32-35.

19. Joshi AD, Pontecorvo MJ, Clark CM, et al. Performance characteristics of amyloid PET with florbetapir F 18 in patients with Alzheimer's disease and cognitively normal subjects. J Nucl Med. 2012;53:378-384.

20. Meyer JH, Gunn RN, Myers R, et al. Assessment of spatial normalization of PET ligand images using ligand-specific templates. Neuroimage. 1999;9:545553.

21. Edison P, Carter SF, Rinne JO, et al. Comparison of MRI based and PET template based approaches in the quantitative analysis of amyloid imaging with PIBPET. Neuroimage. 2013;70:423-433.

22. Landau SM, Breault C, Joshi AD, et al. Amyloid- $\beta$ imaging with Pittsburgh compound $\mathrm{B}$ and florbetapir: comparing radiotracers and quantification methods. J Nucl Med. 2013;54:70-77.

23. Kemppainen NM, Aalto S, Rinne JO, et al. Voxel-based analysis of PET amyloid ligand $\left[{ }^{11} \mathrm{C}\right]$ PIB uptake in Alzheimers disease. Neurology. 2006;67:15751580 . 